\title{
La educación a distancia: características, desafíos y aprendizajes $^{1}$
}

\section{Distance education: characteristics, challenges and learning}

Natalia Andrea Correa Guisao", Lenika Alejandra David Posso", Crisly Vanessa García Quintero ${ }^{* * *}$, Astrid Johana Toro Serna***

Forma de citar este artículo en APA:

Correa Guisao, N. A., David Posso, L. A., García Quintero, C. V., \& Toro Serna, A. J. (2021). La educación a distancia: características, desafíos y aprendizajes. Poiésis, (41), 65-79. https://doi.org/10.21501/16920945.4175

\section{Resumen}

El artículo tiene como finalidad realizar una aproximación y reflexión sobre la educación a distancia en Colombia, rescatando sus principales características, las ventajas y desafíos que enfrenta esta modalidad educativa en la actualidad. El texto se construye con base en una revisión documental y el proceso reflexivo de estudiantes y docentes del semillero de educación social; dicha reflexión puede ser de interés para quienes participan en esta modalidad educativa y quienes gestionan los procesos de educación en el contexto actual.

\section{Palabras clave:}

Colombia; Educación a distancia; Educación virtual; Educación social; Educación; Formación profesional; Trabajo social.

El presente artículo se construye en el marco del proyecto de investigación: "Educación en Trabajo Social a distancia, una reflexión sobre la experiencia del programa de Trabajo Social de la Universidad Católica Luis Amigó", con la participación en el equipo del semillero de investigación en "Educación social y sistematización de experiencias". El proyecto se viene ejecutando desde el año 2019 y finaliza en noviembre de 2020. Asesora: Janeth García Gallego: Trabajadora Social, magíster en Educación, docente del programa de Trabajo Social modalidad distancia, de la Universidad Católica Luis Amigó, coordinadora del semillero "Educación social y sistematización de experiencias". Contacto: Janeth.garciaga@amigo.edu.c0. ORCID: https://orcid.org/0000-0002-0928-3276.

Estudiante del noveno semestre del programa de Trabajo social modalidad distancia, de la Universidad Católica Luis Amigó, integrante del semillero de Educación Social y Sistematización de experiencias, Medellín-Colombia. Contacto: natalia.correagu@amigo.edu.co

Estudiante del noveno semestre del programa de Trabajo social modalidad distancia, de la Universidad Católica Luis Amigó, integrante del semillero de Educación Social y Sistematización de experiencias, Medellín-Colombia. Contacto: Ienika.davidpo@amigo.edu.co

Estudiante del noveno semestre del programa de Trabajo social modalidad distancia, de la Universidad Católica Luis Amigó, integrante de semillero de Educación Social y Sistematización de experiencias, Medellín-Colombia. Contacto: crisly.garciaqu@amigo.edu.co

".*studiante del noveno semestre del programa de Trabajo social modalidad distancia, de la Universidad Católica Luis Amigó, integrante del semillero de Educación Social y Sistematización de experiencias, Medellín-Colombia. Contacto: astrid.torose@amigo.edu.co. 


\section{Abstact}

This article aims to carry out an approach and reflection on distance education in Colombia, describing its main characteristics, the advantages and challenges that this educational modality faces today. The text is constructed based on a documentary review and the reflective process of students and teachers from the social education research field; Such reflection may be of interest to those who participate in this educational modality and those who manage the education processes in the current context.

\section{Keywords:}

Colombia; Education; Long distance education; Social education; Social work; Virtual education; Vocational training. 


\section{Introducción}

En el marco de este ejercicio investigativo se han planteado diversas reflexiones sobre la formación profesional en Trabajo Social desde la modalidad a distancia, y las implicaciones que esto tiene tanto para la comunidad universitaria que influyen en esta, como para la educación misma. En correspondencia con ello, se reconocen los principales conceptos y categorías orientadoras de la investigación, como aporte para la posterior reflexión de la experiencia desarrollada desde el programa de Trabajo social.

Es así como el artículo se organiza de la siguiente forma: en primer lugar se ubica la educación virtual y a distancia en Colombia desde algunas experiencias educativas, al igual que sus aspectos normativos; en segundo lugar, se define la educación a distancia y se exponen sus principales características; finalmente, se plantean oportunidades y los desafíos que enfrenta esta modalidad educativa.

\section{La educación a distancia en Colombia}

La educación a distancia es una modalidad formativa que se ha venido desarrollando a lo largo de la historia como producto de los cambios en el contexto, dadas las necesidades particulares de los territorios y la adaptación de los procesos educativos que han permitido la incorporación de nuevas estrategias y tecnologías de enseñanza y aprendizaje. De acuerdo con Begoña Tellería (2004):

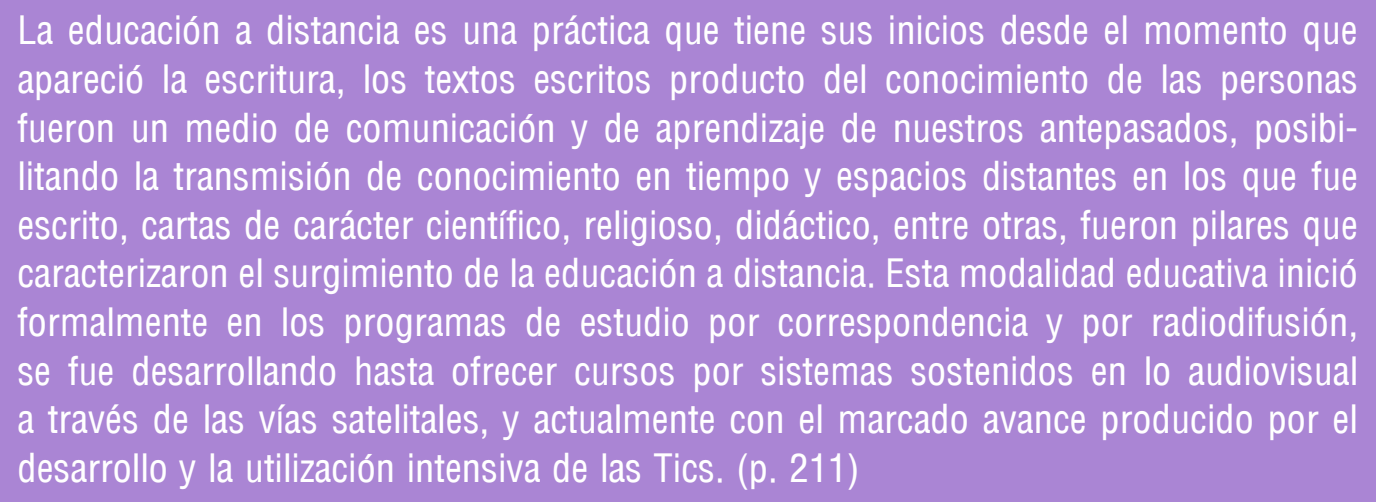

En América Latina, a partir de políticas sociales orientadas al acceso a la educación en países donde las tecnologías de la información y la comunicación no contaban con tanto auge, se crearon diversas estrategias de educación que pretendían formar a la población para la realización de actividades productivas y a nivel profesional, esto tuvo relevancia en el trabajo de las comunidades religiosas que tenían una misión educativa; es así como, 
en 1930 varias escuelas norteamericanas incursionaron en Colombia ofreciendo cursos de capacitación por correspondencia, entre ellos la Acción Cultural Popular (ACPO) que comenzó en 1947 con los cursos a través de radio Sutenza y el seminario El campesino, que tenían como finalidad llegar a los sectores de la población campesina que no contaban con la oportunidad de culminar su proceso de educación. En este momento histórico la educación a distancia se caracterizaba por utilizar medios de comunicación como la radio, al igual que materiales como cartillas, módulos y guías de aprendizaje. (Rodríguez Camargo, 2017, pp. 76-77)

No obstante, "fue durante la década de los 70 que se dio de manera general la Educación a Distancia, pero el término como tal, se acuño luego en el año 1982 por el Consejo Internacional de Educación a Distancia" (García Aretio, 1994, como se cita en García Aretio, 2011, p. 253).

En la segunda mitad del siglo XX, las instituciones de educación superior también se interesaron en aportar a la formación de las personas de sectores rurales. Explica Rodríguez Camargo (2017) que en los años setenta, diversas universidades iniciaron cursos de capacitación a distancia, entre las que se encontraban la Universidad de Antioquia y Universidad Javeriana; ya para la década de los ochenta, durante la presidencia de Belisario Betancur, se presenta el Programa Nacional de Universidad a Distancia, específicamente en el año 1982, el cual pretendía contar con mayor cobertura y menor tiempo para la educación.

Más adelante, en 1997, según los datos del Ministerio de Educación Nacional (como se cita en Rodríguez Camargo, 2017), creció el número de universidades con educación 100\% virtual; en este sentido, la Fundación Universitaria Católica, independiente de la educación a distancia del norte, era la única institución de educación superior que funcionaba bajo esta modalidad; de esta manera, la educación virtual inicia como un modelo.

Por su parte, la Universidad Católica Luis Amigó -en años anteriores denominada Fundación Universitaria Luis Amigó- incursionó en la modalidad de educación a distancia desde el año 1984, a partir de metodologías semipresenciales en lo que llamó centros regionales; y en los años noventa dio un cambio en las metodologías utilizadas con la implementación de "materiales didácticos multimediales elaborados con programas de autor, presentaciones y software educativos que eran instalados localmente en equipos de cómputo" (Arboleda, 2012, p. 303). En la actualidad, los avances didácticos y pedagógicos, el trabajo desde las tecnologías de la información y la comunicación (TIC), la incorporación de plataformas educativas y la formación docente han posibilitado que crezca el número de programas virtuales y a distancia en la Universidad, tanto en el nivel de pregrado como postgrado (Arboleda, 2012).

En Colombia, las modalidades educación virtual y a distancia han crecido y ganado favorabilidad como posibilidad de acceder a la educación superior para las personas que por múltiples razones no pueden hacer parte de la educación presencial; de hecho, en la actualidad muchas universidades utilizan las TIC como apoyo a los programas presenciales y otras ofrecen programas en las tres modalidades (presencial, virtual y distancia), lo cual está soportado en la normatividad vigente que se ha desarrollo en materia de educación en el país. 
Según los avances normativos en materia educativa descritos en el Tabla 1, es relevante mencionar que en 1991 con la nueva Constitución Política se dio un cambio significativo en la concepción de la educación, la cual fue considerada como un derecho fundamental de los colombianos, reiterando así la importancia de una educación basada en la democratización del conocimiento, una educación laica e incluyente. A partir de este cambio normativo surge una nueva legislación que contempla diversas modalidades y metodologías de educación superior.

\section{Tabla 1.}

Normograma

\section{Norma}

Ley 30 de 1992

\section{Finalidad de norma}

Por la cual se organiza el servicio público de la Educación Superior.

\section{Principales consideraciones}

Capítulo III. Artículo 15. Las instituciones de Educación Superior podrán adelantar programas en la metodología de educación abierta y a distancia, de conformidad con la presente Ley.

Artículo 2. Todo programa de educación a distancia propenderá por el desarrollo de competencias requeridas por la metodología, tendrá una estructura curricular flexible y hará explícitas las estrategias de inducción.

Resolución 2755 Por la cual se definen las características Artículo 5. Medios educativos, en donde se incluyen: recursos y políticas de Resolución 2755 específicas de calidad para la oferta y desarrollo, convenios que garanticen las condiciones logísticas e institucionales, desarrollo de los programas académicos laboratorios físicos o virtuales y materiales de apoyo multimedia.

en la metodología a distancia.

Artículo 6. Materiales de los cursos. El programa deberá expresar con claridad las políticas y mecanismos que tiene establecidos para la producción, distribución, evaluación y edición de los materiales propios de los cursos, así como de adquisición de equipos y medios requeridos.

Por el cual se reglamenta el registro

Decreto 1295 de calificado que trata la Ley 1188 de 2008 y

2010 la oferta y desarrollo de programas académicos de educación superior.

Capítulo II. Artículo 5. Evaluación de las condiciones de calidad de los programas a distancia o virtuales, en donde se incluye: idoneidad del personal docente, medios educativos e infraestructura.

Artículo 6. Evaluación de las condiciones de calidad de carácter institucional en donde se incluye: los mecanismos de selección y evaluación, la estructura administrativa y académica y el bienestar universitario para los estudiantes a distancia 0 virtual.

Como puede observarse, la educación a distancia ha ganado validez y se han generado avances fundamentales para su correcto desarrollo, incluyendo asuntos institucionales, curriculares, pedagógicos y tecnológicos, para un adecuado proceso formativo. Esta normatividad, además de generar procesos institucionales, también exige que se realice la inclusión educativa en el marco de condiciones de calidad, con el fin de garantizar un proceso democrático en el acceso a la educación superior en las diferentes modalidades.

\section{Definiciones sobre la educación a distancia}

Con relación al término de distancia, la Real Academia de la Lengua Española (s.f.) describe que proviene del latín distantia y una de sus definiciones es "Que se realiza o funciona por correspondencia 0 a través de los medios de telecomunicación sin que se requiera la presencia física de los participantes" (párr. 9). 
Lo anterior nos lleva a que la educación a distancia puede ser considerada una modalidad educativa que posibilita el acceso a la educación superior de más personas, independientemente del lugar en que se encuentren ubicadas; además, se ha ido reinventando con el paso del tiempo y los cambios tecnológicos, ganando así espacios tanto a nivel nacional como internacional, ya que son cada vez más los programas que se ofrecen en todo el mundo.

Por otra parte, según lo explica Maldonado Juca (2016):

La concepción de la educación a distancia o virtual ha cambiado con el auge de las telecomunicaciones digitales y las tecnologías de la información y la comunicación, esto ha permitido mejores y más rápidas formas para diseminar los conocimientos. Las redes de los sistemas de telecomunicaciones permiten que las personas y grupos tengan conexiones rápidas y confiables lo que ha permitido una mayor interrelación de información entre ellos. La integración de los campos de la informática, los medios audiovisuales y las telecomunicaciones ha causado impacto en la educación a distancia en el contexto de la globalización. (p. 107)

Lo anterior ha supuesto el desarrollo de innovaciones educativas y pedagógicas por parte de las instituciones de educación superior, de tal manera que tanto estudiantes como profesores puedan hacer un uso apropiado de las TIC en los procesos de enseñanza y aprendizaje, al sacar provecho de las conexiones en tiempo real, al igual que del trabajo asincrónico, con ello fomentar el trabajo en equipo y el desarrollo de competencias ciudadanas y laborales necesarias en un mundo interconectado.

Sin embargo, es necesario hacer una diferenciación entre la educación a distancia y la educación virtual. La educación primera requiere de espacios sincrónicos - presenciales y asincrónicos virtuales para el desarrollo del proceso de enseñanza y aprendizaje, es decir, cuenta con escenarios de presencialidad y de ejercicios prácticos contextualizados. Si bien la educación a distancia toma la virtualidad como mediación metodológica, esta no es exclusiva. En cuanto a la educación virtual, ha generado un camino propio, desde modelos y estrategias que definen una propuesta educativa, en la cual no se requiere de la interacción presencial entre profesores y estudiantes, y se depende de las ayudas tecnológicas para la construcción del ambiente educativo y de las relaciones pedagógicas. De ahí que Roldán Cardona y Atehortúa Agudelo (2017) expliquen:

La educación virtual requiere una resignificación del rol que ejercía el docente y el estudiante, se habla entonces de que el papel del docente como reproductor del conocimiento pasará a ser un guía que acompañe el proceso, el papel protagónico en esta modalidad lo llevará el estudiante. (p. 6)

Si bien existen diferencias en estas modalidades educativas, centradas en los espacios de interacción sincrónica entre los actores del proceso educativo, tanto la educación virtual como la educación a distancia fomentan en el estudiante independencia en el proceso de aprendizaje y en la búsqueda de estrategias y herramientas a través de las cuales amplíe la compresión que tienen de 
los problemas o situaciones, y la forma como pueden intervenir en ellas. Así, el estudiante asume un rol activo en su proceso formativo y sus avances dependen del trabajo que realice por cuenta propia a partir de las orientaciones del profesor.

\section{Características de la educación a distancia}

Los programas de educación a través de la virtualidad y en modalidad distancia se reconocen por ser flexibles y cercanos a las necesidades de personas que debido a condiciones geográficas y de inclusión social no pueden acceder a la educación formal presencial. Además, este tipo de educación presenta características que se relacionan con la creación de ambientes de trabajo autónomo y colaborativo, al posibilitar interacciones entre docentes y estudiantes mediadas por las TIC, lo cual contribuye a facilitar aprendizajes contextualizados de acuerdo con realidades regionales y locales. A continuación, se enuncian algunas características de esta modalidad educativa.

\section{Aprendizaje autónomo}

La educación a distancia se caracteriza por desarrollar su metodología desde el trabajo autónomo e independiente por parte de los estudiantes, pero con apoyo del docente en el proceso formativo, denominado por Rodríguez Fernández (2014) como el principio de la autoformación:

Esta se adecua al marco de los principios constructivistas que rigen la educación actual pues supone que la iniciativa y gestión del proceso de aprendizaje está en manos del propio sujeto que aprende, sin excluir por ello la ayuda externa del docente con funciones tutoriales. (p. 80)

Así mismo, en el modelo de autoaprendizaje se reconocen al docente, el estudiante y el contenido y la manera en que se trasmite, como parte esencial al momento de hablar de educación a distancia. Begoña Tellería (2004) plantea que "el contenido se presenta a través del material didáctico, en este tipo de estudio se convierte en un elemento especialmente relevante, ya que en él se incluye toda la información y se ofrece la guía a los alumnos" (p. 211).

Por otra parte, García Aretio (1994) habla del aprendizaje autónomo en términos de independiente y flexible, y hace referencia a que la educación a distancia tiene como fin facultar a los estudiantes en ''aprender a aprender' y 'aprender a hacer' pero de forma flexible, forjando su autonomía en cuanto a tiempo, estilo, ritmo y método de aprendizaje, al permitir la toma de conciencia de sus propias capacidades y posibilidades para su autoformación" (p. 45). De lo anterior podría decirse que el estudiante es el principal sujeto de formación, y es mediante su trabajo que desarrolla habilidades tanto para su proceso académico como para su ejercicio profesional 
El autoaprendizaje como característica de la educación a distancia, permite que los estudiantes ejerzan autonomía y desarrollen la capacidad de tomar decisiones consientes y consecuentes en pro de su proceso educativo. De ahí, la importancia del uso flexible y eficiente del tiempo y el espacio, en aras de fortalecer el autoaprendizaje, de modo tal que se afiancen características como la disciplina y la responsabilidad, se tracen metas y objetivos académicos e implementen estrategias de auto planificación, autorregulación y autoevaluación.

\section{Aprendizaje collaborativo}

El aprendizaje colaborativo implica que los estudiantes, de manera consensuada y mediante la conformación de equipos de trabajo, tracen y logren metas relacionadas con su formación tanto profesional como personal. Se debe tener en cuenta que no está determinado o no significa que los estudiantes realicen trabajos académicos de manera individual y luego junten las partes para obtener un producto final, se requiere lograr metas colectivas que posibiliten el desarrollo de habilidades como la comunicación, la autonomía, el criterio, la disciplina, la responsabilidad y el liderazgo, además, la creación de lazos de confianza.

Si bien se rescata en la educación a distancia el trabajo colaborativo, se pueden dar en sus estudiantes sentimientos poco positivos, ya que el acercamiento a esta modalidad educativa, basada en gran medida en la distancia física y a pesar de estar mediado por las herramientas tecnológicas, que permiten a los estudiantes interactuar, supone para muchos algunas dificultades y retos.

En la educación a distancia es fácil que se produzcan sentimientos de aislamiento y que se pierda la motivación; el sentirse parte de un grupo de aprendizaje ayuda a superar estas dificultades fortaleciendo lazos entre compañeros y tutores que, aunque parecen no ser tan cercanos, terminan convirtiéndose en personas fundamentales en su cotidianidad para el crecimiento personal y profesional. El grupo se convierte en el apoyo afectivo y en el escenario para movilizar diversos recursos (emocionales, académicos, económicos) que permiten continuar el proceso formativo aún en los momentos más complejos; allí se configuran relaciones solidarias, redes de apoyo, identidades y comunidad académica.

Por lo anterior, el trabajo colaborativo va más allá de generar estrategias de aprendizaje y enseñanza dinamizadas por las TIC, y la creación de equipos de trabajo, esto implica también involucrase de manera personal entre estudiantes, y crear así lazos de confianza que posibiliten ejercicios académicos y personales en pro de la consecución de objetivos colectivos. 


\section{Rol del docente - tutor}

Las relaciones pedagógicas son un componente fundamental del proceso educativo, pues a partir de estas se facilita el aprendizaje y la construcción de saberes marcados por la experiencia. En la interacción educativa se encuentran los contenidos, metodologías, las intencionalidades y la pedagogía, es decir, esa forma de construir el conocimiento con el otro; además, en dicho proceso existen roles en los cuales docente y estudiante ocupan lugares diferenciados desde donde aportan en la configuración de la educación misma.

En la educación a distancia, el docente 0 el tutor debe tener presente que su rol es de facilitador y que este no es el tradicional, es decir, el docente visto como el poseedor y trasmisor de conocimiento, sino como un guía que acompaña, direcciona y posibilita el aprendizaje autónomo por parte del estudiante. En palabras de Rodríguez Camargo (2017) son "lideres tutores que estimulan y orientan los procesos de autoaprendizaje de los estudiantes" (p. 99).

La educación en general tiene retos para los docentes, ya que cada vez son más las exigencias del medio, los avances de la tecnología, incluso, podría decirse que muchos estudiantes son inquietos por el conocimiento y esto pone al docente en el lugar de la constante actualización de sus propuestas pedagógicas y didácticas, pero "en la modalidad a distancia esta continua actualización se hace imprescindible desde todo punto, dado el avance de las tecnologías de la información y comunicación, esenciales en los procesos de formación a distancia" (García Aretio, 1994, p. 295).

Esta labor de facilitar y acompañar el proceso formativo implica la construcción de estrategias para crear el vínculo educativo, interacciones cotidianas facultadas por las TIC, en las cuales se construye una relación pedagógica de conocimiento y reconocimiento que no depende únicamente del encuentro físico, sino de la interacción mediada.

\section{Sentido de presencia en las relaciones pedagógicas}

Las relaciones pedagógicas en los ambientes de aprendizaje propician espacios de confianza tejiendo relaciones interpersonales entre docentes y estudiantes, lo que posibilita un apropiado desarrollo de la formación a nivel individual y grupal. A su vez, el sentido de presencia que se establece en el grupo determina elementos importantes que permiten el desarrollo de la actividad de enseñanza-aprendizaje.

Para entender las relaciones pedagógicas, exponen Jurado Mejía y Obando Sánchez (2014) que se deben tener en cuenta diferentes perspectivas o modelos pedagógicos, tales como el tradicional, el conductista, el cognitivo, el romántico y el social. Los tres primeros asociados a la verticalidad, es decir, el profesor como poseedor del conocimiento y el estudiante como receptor del mismo. En cambio, las dos últimas, se corresponden con la horizontalidad en la relación, es decir, una relación en la que tanto docentes como estudiantes pueden producir conocimiento a través de las interacciones. 
Siguiendo con Jurado Mejía y Obando Sánchez (2014) como referentes, ellos plantean que la relación pedagógica va más allá de estas dos visiones y que las nuevas tendencias están marcadas por el uso de las TIC, esto es:

Relaciones pedagógicas son aquellas que se dan entre profesor y estudiante durante el acto educativo. Se sospecha una modificación en su carácter comunicativo al ser mediadas por las TIC, es decir, su democratización, que promete alcanzar mayor intensidad si se comprende que no es un asunto meramente técnico. (p. 57)

Por otra parte, de la mano de Lehman y Conceição (2010) se plantea la presencia desde dos fenómenos: "la telepresencia en el entorno en línea ocurre cuando los alumnos tienen la impresión 0 la sensación de estar presentes en un lugar alejado de su propio entorno inmediato. Presencia social significa interacciones con otros en el entorno online" (p. 3). Es decir, el docente y los estudiantes hacen presencia tanto en términos de estar en el entorno virtual de aprendizaje (ingresar a la plataforma tecnológica), como de la interacción con los otros.

Entender el sentido de presencia en el ambiente social de aprendizaje brinda ventajas tanto para docentes como para estudiantes. La primera, las actividades de enseñanza-aprendizaje se vuelven significativas para los actores involucrados, puesto que ese significado no solo es personal sino también socialmente construido. Segunda, se disminuye el sentimiento de aislamiento del estudiante, pues el concepto va más allá de la tele presencia al incluir el aspecto social y experiencial. Tercera, permite un diseño aterrizado de las actividades, pues tiene en cuenta los momentos antes, durante y después de las mismas. Cuarta, este concepto reconoce que el uso de las tecnologías no es suficiente.

\section{Aprendizaje contextualizado}

Cabe resaltar que la educación a distancia posibilita la inclusión social en contextos diversos, donde los estudiantes cuentan con una caracterización específica, siendo los mayores beneficiados jóvenes y adultos que tienen responsabilidades laborales o familiares que limitan su tiempo de estudio, personas con discapacidad, población distante de las sedes universitarias o con condiciones sociales, económicas o geográficas diversas; lo que permite el acceso a la educación superior y conlleva a mayores posibilidades de mejorar su calidad de vida, nivelación laboral o superación personal.

El aprendizaje contextualizado se refiere a los procesos de enseñanza aprendizaje en los cuales se realiza una lectura del contexto y se construyen propuestas que, desde lo académico, pueden aportar en la comprensión e intervención de los fenómenos sociales particulares desde los diversos programas académicos. Si bien esta es una exigencia de la educación en general, en la educación virtual y a distancia se hace más importante por la diversidad de sujetos y, por ende, de contextos que confluyen en el proceso formativo. 
En este sentido, el aprendizaje contextualizado reconoce los entornos y los pone en diálogo constante con los contenidos y metodologías, además posibilita el diálogo de saberes entre los estudiantes que se encuentran en contextos diversos, facilitando con esto una mirada amplia de la realidad nacional y la comprensión de posibles formas de intervención.

\section{Uso de metodologías alliernativas e innovadoras}

Las TIC se presentan como una alternativa para dar respuesta a demandas de educación superior. Posibilitan el acceso al campo académico, fomentando espacios de inclusión, flexibilidad y accesibilidad a través de la modalidad de educación a distancia, ofertada por diferentes instituciones de educación superior del país, donde los estudiantes están inmersos en una dinámica no tradicional, ni convencional, pero aun así se tiene la posibilidad de explorar y potencializar habilidades con uso de las diferentes tecnológicas que facilitan la relación e interacción educativa.

Las TIC propician la comunicación, la interacción y la transferencia de la información a través de diversas herramientas tecnológicas, entre las cuales se pueden mencionar plataformas digitales, foros, chats, correo electrónico, video conferencia o video llamada, tutorías sincrónicas o asincrónicas y bases de datos digitales; esto sumado a los contenidos propuestos por el docente y la capacidad de autoaprendizaje de los estudiantes. Estas herramientas por sí solas no crean el ambiente de enseñanza y aprendizaje; adquieren relevancia en los diferentes módulos guías, textos, así como en las interacciones que permiten el desarrollo del proceso académico.

\section{Oportunidades y desafíos de la educación a distancia}

Según lo planteado en el presente texto, la educación virtual y a distancia tiene oportunidades y retos que se relacionan con los desafíos que en general tiene la educación, algunos de estos se señalan a continuación.

En cuanto a las oportunidades, en el plano individual, la educación a distancia posibilita el autoaprendizaje desde el desarrollo de la autonomía y la disciplina de estudio, además de la capacidad de planeación de las actividades sin depender del trabajo en clase con el docente; por ser una modalidad en la que se trasciende el espacio tiempo educativo, también tiene como bondades la accesibilidad, la facilidad de horarios y la reducción de costos educativos.

La utilización de estrategias tecnológicas permite la conectividad con otras formas de comprender y construir conocimiento, con contextos y problemáticas diversas, lo que hace que en este tipo de modalidades se pueda acceder a escenarios múltiples de la realidad social, los cuales hacen parte de los contenidos de estudio, generando desde allí procesos de integración y construcción de conocimiento anclados en el contexto social. 
Es un proceso basado en la innovación tecnológica, que pone a prueba las capacidades de los estudiantes para adaptarse a las tecnologías que permiten su educación. Además, las mediaciones tecnológicas hacen que el proceso sea dinámico, facilitando otras formas de interacción sincrónicas, cara a cara o virtuales. Es lo que sucede con el acceso al conocimiento desde la esfera digital por medio de conexión a internet, también por medio de juegos y plataformas educativas que se pueden instalar en los dispositivos digitales. Se destaca una democratización en el uso y acceso a la información, en la que se pueden encontrar múltiples recursos que facilitan, amplían, expanden el conocimiento, ofreciendo oportunidades puntuales a las personas que, por sus condiciones sociales, económicas, geográficas, no pudieran ser parte de un proceso de educación presencial (Roldán Cardona \& Atehortúa Agudelo, 2017, p. 6).

Por su parte, los desafíos que enfrenta la educación a distancia tienen que ver con los recursos necesarios para llegar de una forma adecuada, con calidad y oportunidad a las personas para las cuales se está proyectando, brindar el acompañamiento pedagógico requerido para la educación superior, poner los procesos educativos al servicio de las comunidades e innovar en los procesos y metodologías de aprendizaje.

Hasta aquí se han abordado los desafíos que tiene la educación en general, no solo la educación virtual y a distancia, lo cual hace relevante nombrar algunas dificultades particulares que enfrentan estas modalidades. La primera de ellas tiene que ver con el poco reconocimiento de estas propuestas como posibilidades de formación válidas y necesarias para la educación en diferentes lugares del territorio nacional; y la segunda, con una mirada conservadora que pone como ideal la educación presencial.

\section{Como lo plantea Mena (2014):}

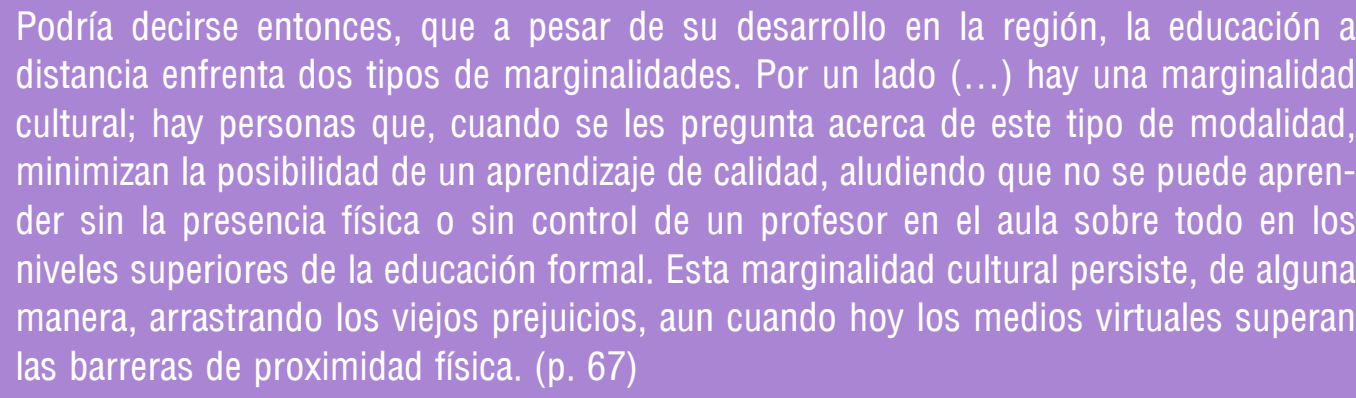

Por el otro, hay una marginalidad jurídica; la legislación educativa no acompañó ni promovió el desarrollo de la modalidad en una región donde podría haber resuelto grandes problemas de aislamiento, de distancias o de exclusión.

Uno de los argumentos ligados a la marginalidad cultural tiene que ver con la interacción que se da en el proceso educativo a distancia o mediado por la virtualidad. Tradicionalmente puede creerse que en estos espacios no se construyen procesos interactivos que posibiliten el aprendizaje. 
Es importante reconocer que la interacción en los procesos educativos virtuales y a distancia se da a través de la mediación de las tecnologías de la información y comunicación, desde las cuales es posible la comunicación sincrónica, la escucha y visualización de la otra persona, donde se plantean unas condiciones importantes para que pueda configurarse una interacción válida y significativa en el proceso educativo.

En la red se pueden callar muchas cosas; el contacto humano puede ser posibilitador para que el estudiante se apasione, se interese por temas particulares por medio de la explicación de un maestro; la interacción cara a cara permite conocer más detalles del proceso en el cual un educador despliega unas acciones sobre el otro y puede dar detalle de las reacciones del mismo (Roldán Cardona \& Atehortúa Agudelo, 2017, pp. 7-8). De ahí que no pueda negar la importancia de la interacción cara a cara y los procesos prácticos para la formación profesional, hay cosas allí que no podrán ser aprendidas desde una mediación virtual, por ello la educación a distancia ofrece una importante combinación entre las mediaciones virtuales y la presencialidad, el trabajo in situ y los procesos prácticos.

Reconocer estas modalidades de educación como posibilidades diferentes a la educación presencial implica también un cambio de paradigma y una apertura a los procesos educativos en la actualidad, para no seguir validando la efectividad de estas propuestas con la educación presencial como unidad de medida. Ello envuelve apertura, reconocimiento y establecer condiciones por parte de quienes ofrecen estas modalidades.

Suele ser común que la evalúen comparándola con la modalidad presencial o utilizando las mismas pautas 0 estándares. Es en este sentido que resulta imprescindible que las agencias de acreditación de toda la región planteen marcos regulatorios que no solo regulen y evalúen la modalidad en forma pertinente, sino que además promuevan y potencien su desarrollo (Mena, 2014, p. 67).

Los procesos de educación en la actualidad han mostrado la diversificación de la educación, en espacios, metodologías, técnicas y mediaciones; sin embargo, la educación como derecho y la importancia de esta para el desarrollo de las sociedades es un consenso que permanece en los países democráticos y se hace explícito en las políticas educativas; en concordancia con lo anterior, es importante conocer las modalidades educativas distancia - virtual a profundidad y analizar sus posibilidades como opción viable en la educación actual, sin marginalizar esta educación y sin construir mesianismos sobre las mismas, valorando los aportes que desde allí se realizan para una educación con calidad, incluyente, contextualizada y transformadora para todas y todos. 


\section{Conflicto de intereses}

Las autoras declaran la inexistencia de conflicto de interés con institución o asociación comercial de cualquier índole.

\section{Referencias}

Arboleda, H. (2012). Hitos de la educación a distancia: una experiencia de la Fundación Universitaria Luis Amigó. Blog Buenas Tareas. https://www.buenastareas.com/ensayos/Hitos-De-LaEducaci\%C3\%B3n-a-Distancia/6498495.html

Begoña Tellería, M. (2004). Educación y nuevas tecnologías. Educación a Distancia y Educación Virtual. Revista de Teoría y Didáctica de las Ciencias Sociales, (9), 209-222.

García Aretio, L. G. (1994). Educación a distancia hoy. Universidad Nacional de Educación a Distancia - UNED.

García Aretio, L. (2011). Perspectivas teóricas de la educación a distancia y virtual. Revista Española de Pedagogía, (249), 255-271. https://reunir.unir.net/bitstream/handle/123456789/3835/ perspectivas $\% 20$ teoricas.pdf?sequence $=1$

Jurado Mejía, V., \& Obando Sánchez, G. (2014). Una aproximación a las relaciones pedagógicas mediadas por las tecnologías en la Institución Educativa Juan Manuel González (Colombia). Páginas: Revista Académica e Institucional de la UCPR, (96), 53-68. https://revistas. ucp.edu.co/index.php/paginas/article/view/1905/1759

Lehman, R., \& Conceição, S. (2010). Creating a sense of presence in online teaching: how to "be there» for distance learners. Jossey-Bass.

Maldonado Juca, F. (2016). La educación a distancia, una necesidad para la formación de los profesionales. Revista Universidad y Sociedad, 8(1), 106-111.

Mena, M. (2014). La educación a distancia: prejuicios y desafíos de la modalidad. Virtualidad, Educación y Ciencia, 5(8), 66-73. https://revistas.unc.edu.ar/index.php/vesc/article/view/7476 
Rodríguez Camargo, M. (2017). Análisis de la gestión educativa en educación a distancia con mediación virtual en educación superior en Colombia [Tesis Doctoral, Universidad de Granada]. Repositorio Digibug. https://digibug.ugr.es/bitstream/handle/10481/45894/26504820. pdf? sequence $=6$ \&isAllowed $=\mathrm{y}$

Rodríguez Fernández, N. (2014). Fundamentos del proceso educativo a distancia: enseñanza, aprendizaje y evaluación. RIED. Revista Iberoamericana de Educación a Distancia, 17(2), 75-93. https://doi.org/10.5944/ried.17.2.12679

Roldán Cardona, J., \& Atehortúa Agudelo, R. (2017). La educación virtual un campo para el análisis: ventajas y desventajas. Docplayer. https://docplayer.es/58112647-La-educacion-virtual-uncampo-para-el-analisis-ventajas-y-desventajas-la-educacion-virtual-un-campo-para-el-analisis-ventajas-y-desventajas.html 